\title{
Design for Several Hydraulic Parameters of a Quadruped Robot
}

\author{
Xuewen Rong ${ }^{1, *}$, Yibin $\mathrm{Li}^{1}$, Jian Meng ${ }^{1}$ and Bin $L I^{2}$ \\ ${ }^{1}$ School of Control Science and Engineering, Shandong University, Jinan, 250061, China \\ ${ }^{2}$ School of Science, Qilu University of Technology, Jinan, 250353, China
}

Received: 14 Sep. 2013, Revised: 12 Dec. 2013, Accepted: 13 Dec. 2013

Published online: 1 Sep. 2014

\begin{abstract}
For a quadruped robot with 12 active joints driven by linear hydraulic actuators, some important parameters, such as the oil flow of the hydraulic system and the needed maximum output force of each actuator, are hard to calculate precisely with kinematics and dynamics equations. The oil flow is mainly determined by the walking speed of the quadruped robot, but also influenced by the stride frequency, gait type and the foot trajectory. This paper planned the foot trajectory with cubic polynomial firstly. Then selected optimal stride frequency referred to that of four-legged mammals and stride length according to the required walking speed of the quadruped robot. Furthermore, displacement curves and velocity curves with respect to time for all linear hydraulic actuators can be obtained easily with inverse kinematics equations in MATLAB. The needed maximum output force of each actuator can be obtained from dynamic simulation with MSC.ADAMS. Then the effective area of the hydraulic actuator can be calculated according to the output force of actuator and oil pressure of the hydraulic system. Lastly, the oil flow can be calculated precisely by summing all the products of the velocity and the effective area of each actuator.
\end{abstract}

Keywords: Hydraulic system, MATLAB, Quadruped Robot, Stride Frequency

\section{Introduction}

Mobile robots mainly include wheeled robots, tracked robots, legged robots and those with other locomotion patterns. Wheeled robots can travel very fast, but they usually require fairly flat floor over which to work. Tracked robots can move in rougher terrain, but slower than wheeled robots. Legged robots are superior to wheeled and tracked robots since they have higher terrain adaptivity, possess greater mobility and flexibility, do little damage to the environment, etc. Nevertheless, legged robots are more complicated in structure and difficult to control than wheeled and tracked robots. The theories concerned with legged robots are still in the development stage.

During the last four decades, walking (or legged) robots have been an exciting research field. Many improvements have been made since the first full computer-controlled robot Phoney Pony was made in University of Southern California in 1966 [1]. Marc Raibert has successfully built some legged robots with one, two or four legs respectively and achieved dynamic running firstly in the world in 1980s [2]. Since then, a large number of walking robots, such as BISAM [3],WARP1 [4], KOLT [5], Tekken [6], HuboDog [7] and etc, have been developed all over the world.

Compared with electric motor driving system, hydraulic driving system has several important advantages such as higher power-to-mass ratio, greater endurance and refueling conveniently, faster dynamic response, better load stiffness and etc. Boston Dynamics Company made their new quadruped robot named BigDog public in 2006 firstly [8]. It is a hydraulic actuated robot with the highest performance that has been never achieved before. Soon after that, the researchers of Korea Institute and Technology and University of Genoa made their hydraulic actuated robot name p2 and HyQ public respectively $[9,10]$.

The authors of this paper have done lots of work about mechanical structure, kinematics analysis and dynamics simulation for hydraulic actuated quadruped robot [11, 12]. The Robotics Research Center of Shandong University has built a quadruped robot in 2010, whose three dimensional model is shown in Fig. 1. It weighs about $65 \mathrm{~kg}$ without hydraulic power pack and can run at

\footnotetext{
* Corresponding author e-mail: rongxw@ @ sdu.edu.cn
} 
the velocity of $1.8 \mathrm{~m} / \mathrm{s}$ with trot gait on flat ground inside laboratory powered by a stationary hydraulic station.

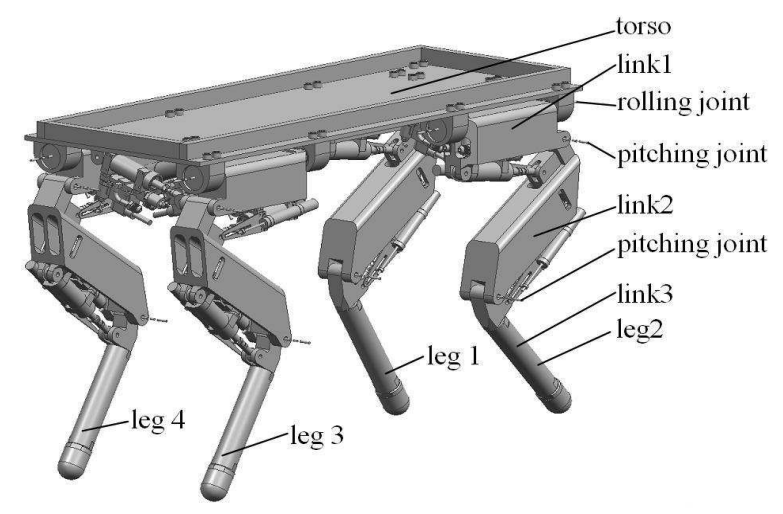

Fig. 1: Three dimensional model of the quadruped robot.

As shown in Fig. 1, each leg of the robot has a rolling rotary joint and two pitching rotary joints. All 12 joints are actuated by linear hydraulic servo cylinders, each of which is composed of one single rod cylinder, one servo valve, one displacement and two pressure sensors.

\section{FOOT TRAJECTORY PLANNING}

The impact forces at the touchdown moment affect the stability of the quadruped robot greatly. In order to minimize the impact forces between ground and the landing foot, the foot trajectory should meet the demand that velocity becomes to zero at the moment of touchdown, lift-off and maximum foot height. The composite cycloid foot trajectory meets the demand well [13]. But it has a serious defect that is the obvious relative slippage occurred between feet and floor at landing moment. The defect has been verified in experiments with physical robot. Kyeong Yong Kim and Jong Hyeon Park propose a new ellipse-based trajectory generation method for galloping quadruped robot [14]. The center of gravity of a quadruped robot will fluctuate greatly along forward and vertical directions while it runs using ellipse foot trajectory.

This paper gives a composite curve trajectory composed of cubic polynomial curve and straight line. The foot trajectory meets the requirements that the velocities of the feet becomes to zero at the moment of touchdown and lift-off and there are no fluctuations with the velocities of the torso along $z$ and $x$ axes. The equations for foot trajectory can be assumed as below:

$$
\begin{aligned}
& x_{s w}(t)=L \times\left(A_{x} t^{3}+B_{x} t^{2}+C_{x} t+D_{x}\right) \\
& z_{s w}(t)=H \times\left(A_{z} t^{3}+B_{z} t^{2}+C_{z} t+D_{z}\right)
\end{aligned}
$$

$$
\begin{gathered}
x_{s t}(t)=L \times\left(K_{x} t+E_{x}\right) \\
z_{s t}(t)=0
\end{gathered}
$$

where, $t$ represents time within one cycle, $L$ represents stride length, $T$ represents cycle time, and $H$ represents the maximum foot height. $A_{x}, B_{x}, C_{x}, D_{x}, A_{z}, B_{z}, C_{z}, D_{z}$, $K_{x}$ and $E_{x}$ are unknown coefficients to be solved. Eqs. (1) and (2) are for swing stage. Eqs. (3) and (4) are for stance stage.

The initial conditions for the foot trajectory equations with respect to torso fixed coordinate frame are listed as follow:

$$
\begin{gathered}
x_{s w}(0 T)=-\frac{L}{4} \\
x_{s w}(0.5 T)=\frac{L}{4} \\
x_{s t}(0.5 T)=\frac{L}{4} \\
K_{x}=-\frac{L}{T} \\
\dot{x}_{s w}(0 T)=K_{x} \\
\dot{x}_{s w}(0.5 T)=K_{x} \\
z_{s w}(0 T)=0 \\
z_{s w}(0.25 T)=H \\
z_{s w}(0.5 T)=0 \\
\dot{z}_{s w}(0.25 T)=0
\end{gathered}
$$

Substituting these initial conditions into Eqs. (1) to (4), all the unknown coefficients can be solved. Then the foot trajectory equations with respect to torso fixed coordinate frame for swing stage are obtained as follow:

$$
\begin{gathered}
x_{s w}(t)=L \times\left(-\frac{16}{T^{3}} t^{3}+\frac{12}{T^{2}} t^{2}-\frac{1}{T} t-\frac{1}{4}\right) \\
z_{s w}(t)=H \times\left(-\frac{128}{T^{3}} t^{3}+\frac{48}{T^{2}} t^{2}\right)
\end{gathered}
$$

The foot trajectory equations with respect to torso fixed coordinate frame for stance stage are

$$
\begin{gathered}
x_{s t}(t)=L \times\left(-\frac{1}{T} t+\frac{1}{4}\right) \\
z_{s t}(t)=0
\end{gathered}
$$




\section{DYNAMICS SIMULATION}

The quadruped robot is a complex multi-body dynamic system. The driving forces of the hydraulic cylinders and contact forces from ground can not be calculated accurately. Dynamics simulation is an effective and convenient way to get them. MSC.ADAMS is a famous dynamics simulation software for multi-body mechanical system in the world. The virtual prototype of the quadruped robot in MSC.ADAMS is shown in Fig. 2 [15].

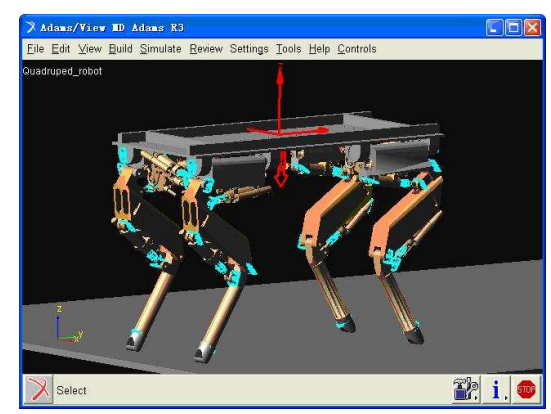

Fig. 2: Virtual prototype of the quadruped robot in MSC.ADAMS.

There are two commonly simulation methods to implement complex motion trajectory in MSC.ADAMS software. One is to plan complex foot motion trajectory in MATLAB or other softwares. Then to generate the joints driving motions for rotary or linear actuators according to the inverse kinematic equations. The joints driving motions are saved as discrete data into a text file. Next, the discrete data is imported into MSC.ADAMS to generate some splines which will be set to rotary or linear driving motions for all active joints with function CUBRSPL(). The other method is to do co-simulation with MSC.ADAMS and MATLAB softwares. The second method can implement the closed loop control for all active joints and is convenient to adjust any parameters to obtain more simulation results. In the initial development stage of the quadruped robot, the purpose of simulation is to get some important parameters, such as joint driving forces and contact forces with the ground. No control algorithm and architecture are considered, so the first simulation method is selected.

Stride frequency is an important specification of the quadruped robot, which plays an important role on the dynamic stability of the robot. Running with higher stride frequency, the robot is more stable. But it demands the hydraulic cylinders have high dynamic response frequency and larger oil flow. Heglund and Taylor have studied that how speed and stride frequency change with body size [16,17]. Stranga and Teudel have done the similar work [18]. The studies show that the perfect stride frequency for quadrupeds with body size from $20 \mathrm{~kg}$ goats to $680 \mathrm{~kg}$ horses ranges from $3 \mathrm{~Hz}$ to $2 \mathrm{~Hz}$ correspondingly.

Fig. 3 shows the simulink model of the quadruped robot. In the simulations, the cycle time is set to 0.4 second, the stride length is set to $400 \mathrm{~mm}$, the maximum foot height is set to $50 \mathrm{~mm}$ and the digital clock frequency is set to $200 \mathrm{~Hz}$ respectively. Trot gait and cubic polynomial are selected as the locomotion gait and foot trajectory. The motions of the four feet and twelve hydraulic cylinders are planned in two MATLAB functions. The displacement and velocity variation curves of twelve hydraulic cylinders are output to "Simout" matrix with discrete data format. Then export the displacement variation curves from "Simout" matrix to a text file and import to MSC.ADAMS to generate twelve splines for active joints correspondingly. The simulation step size can be set to 0.005 second or larger.

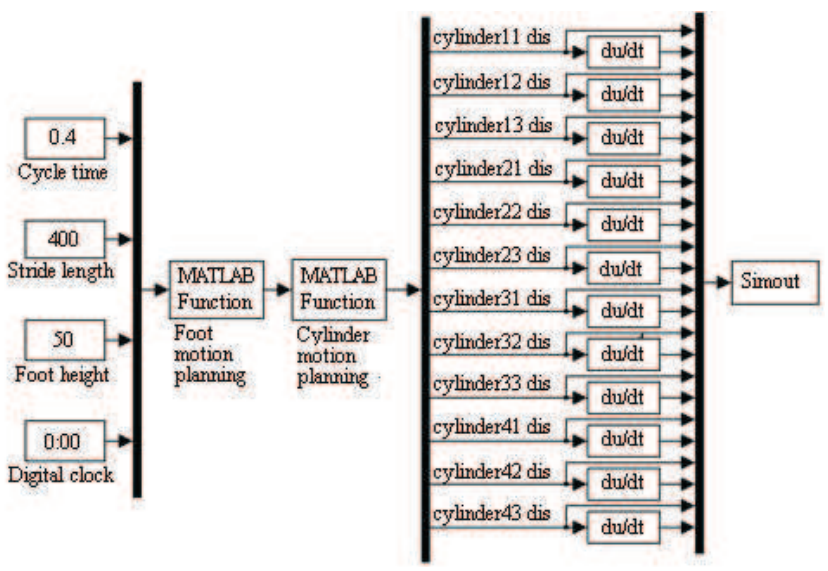

Fig. 3: Simulink model of the quadruped robot.

The output forces variations during one cycle time of three hydraulic cylinders of one leg are shown in Fig. 4, from which it can be drawn that the maximum driving force is about $4200 \mathrm{~N}$ for the three actuators. Since the four legs have identical mechanical structure, so it can be considered as the maximum driving force for all twelve hydraulic cylinders. It provides an important reference for the design of the hydraulic cylinders. Under the assumption that the hydraulic system pressure is set to 21 $\mathrm{MPa}$, the best effective load pressure drop of the servo valve is $14 \mathrm{MPa}$ for the actuator has the highest efficiency with this pressure drop [19]. So, the smallest effective area of the hydraulic cylinder can be determined as 300 $\mathrm{mm}^{2}$, which can be considered as the area of piston end with rod. Further, the piston and rod diameters of the cylinder can be initially determined to $22 \mathrm{~mm}$ and $10 \mathrm{~mm}$ respectively. 


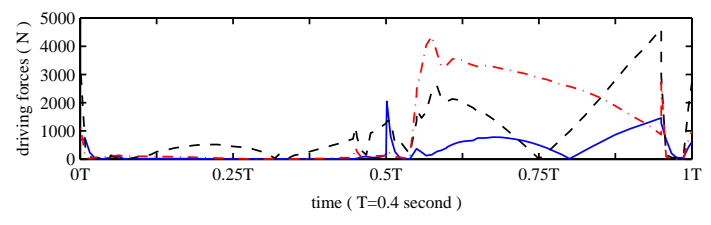

Fig. 4: Driving forces of three hydraulic cylinders of one leg in simulation with the total weight of the quadruped robot is set to about $200 \mathrm{~kg}$.

\section{HYDRAULIC PARAMETERS CALCULATION}

\subsection{Hydraulic system oil flow rate calculation}

The hydraulic system oil flow rate is an important parameter, which varies with the running velocity of the quadruped robot. It is also affected by the locomotion gait, stride length and stride frequency of the robot. For a single hydraulic servo cylinder, the oil flow rate is determined by the velocity and effective area of the piston and the internal leakage of the servo valve. The leakage of the cylinder is ignored.

Running at high speed on flat ground needs larger oil flow rate for the quadruped robot. While it runs on uneven terrain, obviously the speed is slower and the oil flow rate is less. The quadruped robot is mainly running straightly at high speed, so the four cylinders for rolling joints of four legs are seldom extending or retracting at this case. Here, a oil flow rate calculation method is given under the assumption that the quadruped robot is running straightly with trot gait and other parameters are set identical to the simulink model as shown in Fig. 3. The velocity variations of eight cylinders for pitching joints of four legs are shown in Fig. 5 respectively. In order to get the oil flow rate of the eight cylinders conveniently, the eight velocity curves can be superposed to one as shown in Fig. 6.

From Fig. 6 and the simulation result of simulink model shown in Fig. 3, it can be drawn that the average and maximum velocities of eight cylinders during one cycle time are $785 \mathrm{~mm} / \mathrm{s}$ and $1106 \mathrm{~mm} / \mathrm{s}$. It can also be drawn that the average velocity at $868 \mathrm{~mm} / \mathrm{s}$ during 0 to 0.25 cycle time is larger than $702 \mathrm{~mm} / \mathrm{s}$ during 0.25 to 0.5 cycle time. Assuming the piston and rod diameters of the cylinder are $22 \mathrm{~mm}$ and $10 \mathrm{~mm}$ respectively. Then the average oil flow rate of the eight cylinders can be calculated as follow:

$$
Q_{1}=v_{a} \cdot A
$$

where $v_{a}$ represents the average superposed velocity of eight cylinders and equals to $785 \mathrm{~mm} / \mathrm{s}, A$ represents the piston area and equals to $380 \mathrm{~mm}^{2}$.

The internal leakage of servo valve is given by its producer. The internal leakage for the first and second (a)

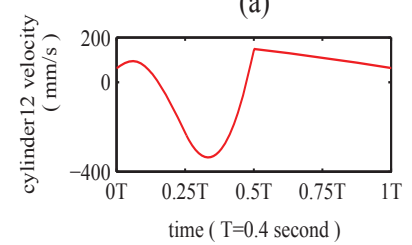

(c)

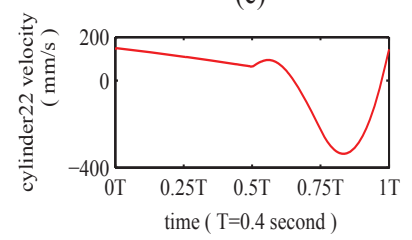

(e)

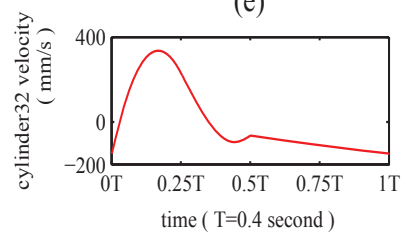

(g)

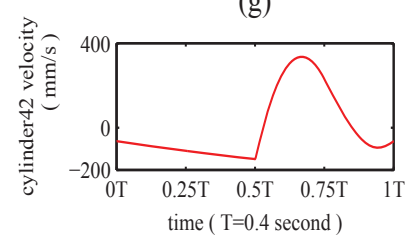

(b)

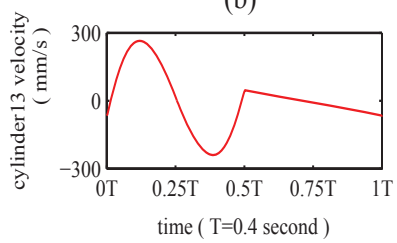

(d)

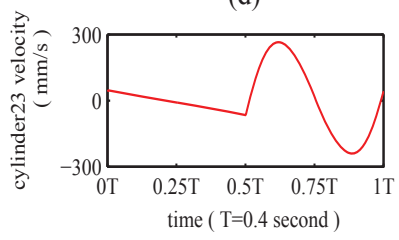

(f)

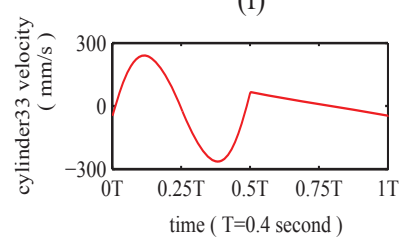

(h)

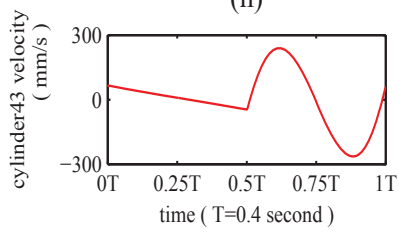

Fig. 5: Velocity variations of eight cylinders during one cycle time.

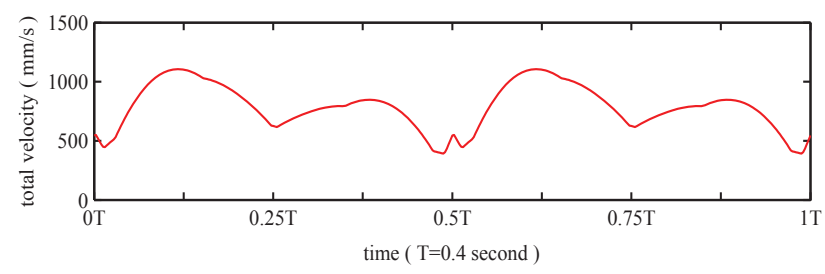

Fig. 6: Superposition of velocity variations of eight cylinders during one cycle time. The extending velocity is positive and the retracting velocity is converted to extending velocity equivalently according to the area ratio between two ends of the piston.

stage are $0.5 \mathrm{~L} / \mathrm{min}$ and 4 percent of the rated flow of the selected servo valve respectively. From Fig. 5 it can be known that the maximum velocity of the eight cylinders is $336 \mathrm{~mm} / \mathrm{s}$. Thus, the rated flow of one servo valve can be determined by

$$
Q_{n}=Q_{i l}+v_{c m} \cdot A
$$

where $v_{c m}$ represents the maximum velocity of eight cylinders and equals to $336 \mathrm{~mm} / \mathrm{s}, Q_{i l}$ represents the 
internal leakage for the first stage of servo valve and equals to $0.5 \mathrm{~L} / \mathrm{min}$.

All twelve servo valves exist internal leakage at the first stage and the four servo valves for the rolling joints exist internal leakage at the second stage. They can be calculated as below:

$$
\begin{gathered}
Q_{2}=12 Q_{i l} \\
Q_{3}=4 \times 0.04 Q_{n}
\end{gathered}
$$

The total oil flow rate of the quadruped robot is determined by

$$
Q=Q_{1}+Q_{2}+Q_{3}
$$

From Eq. (20), we obtain

$$
Q_{n}=8.16
$$

Referring to the servo valve specifications from its producers, the servo valve with rated flow at $10 \mathrm{~L} / \mathrm{min}$ is selected. Thus, the average oil flow rate of the quadruped robot can be obtained as below:

$$
Q=25.5
$$

\subsection{Accumulator volume calculation}

It can be seen from Fig. 6 that the total oil flow rate of the quadruped robot does cyclically changes with a cyclic period of 0.2 second. It can be calculated that the average oil flow rate during the first and second half cycles are $27.4 \mathrm{~L} / \mathrm{min}$ and $23.6 \mathrm{~L} / \mathrm{min}$ respectively. In order to reduce the power and weight of the hydraulic power pack and void overheating, the output oil flow of the hydraulic pump should be adjusted to equal to or slight larger than the average oil flow of the entire cycle. So a hydraulic accumulator is necessary to store high pressure oil during the second half cycle and output high pressure oil during the first half cycle of the next period. The total volume of the hydraulic accumulator can be calculated with the following equation [20]:

$$
V_{0} \geq \frac{V_{w}\left(p_{1} / p_{0}\right)^{\frac{1}{n}}}{1-\left(p_{1} / p_{2}\right)^{\frac{1}{n}}}
$$

where $V_{0}$ represents the total volume of the accumulator, $V_{w}$ represents the working volume, $p_{0}$ represents the gas filling pressure, $p_{1}$ and $p_{2}$ represent the lowest and highest pressure in working respectively, $n$ is a coefficient concerned with the speed of heat conducting.

According to the average oil flow rates in two half cycles, it can be calculated that the working volume $V_{w}$ of the hydraulic accumulator is $0.0032 \mathrm{~L}$. Generally, the gas filling pressure $p_{0}$ is less than 90 percent of $p_{1}$ and larger than 25 percent of $p_{2}$. It can be set to $12 \mathrm{MPa}$. The highest pressure $p_{2}$ is set to $21 \mathrm{MPa}$. In order to reduce the pressure fluctuations, the lowest pressure $p_{1}$ is set to $20 \mathrm{MPa}$. Since the accumulator works at high frequency, its working procedure can be considered as a adiabatic process. The coefficient $n$ should be assigned as 1.4. So the total volume of the accumulator can be obtained as

$$
V_{0} \geq 0.135
$$

The working volume varies greatly with the stride frequency of the quadruped robot. With the same theoretical running speed, the working volume calculated from Fig. 7. is about 2 times of that from Fig. 6. Therefore, the actual volume of the hydraulic accumulator should be selected larger than the calculation value.

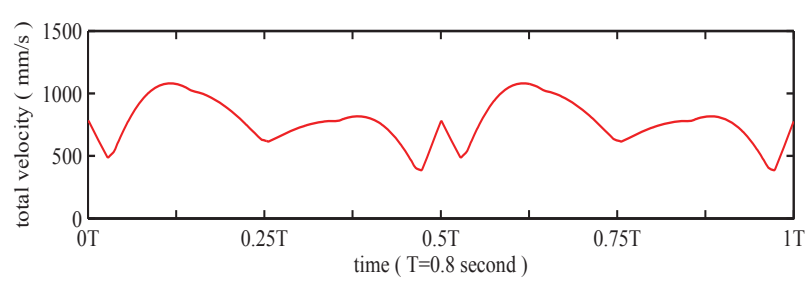

Fig. 7: Superposition of velocity variations of eight cylinders during one cycle time. In this simulation, the cycle time is set to 0.8 second and the stride length is set to $800 \mathrm{~mm}$. The theoretical running velocity of the robot is $1 \mathrm{~m} / \mathrm{s}$ also.

\section{Conclusion}

This paper presents a quadruped robot configuration driven by linear hydraulic servo cylinders firstly. Then a composite foot trajectory composed of cubic polynomial and straight line is given. Further, dynamics simulation is made for obtaining the driving forces of three hydraulic cylinders of one leg. Finally, the oil flow of the robot and the volume of the accumulator are calculated precisely.

The results of simulations in this paper provide important reference for the development of the quadruped robot. In the following work, we will design the miniaturized hydraulic power pack driven by an internal combustion engine, develop dynamic stability control algorithm and do lots of experiments on outdoor uneven terrain.

\section{Acknowledgement}

This work is supported by National Natural Science Foundation of China (61233014, 61305130), Shandong Provincial Natural Science Foundation (ZR2013FQ003, 
ZR2013EEM027) and Independent Innovation Foundation of Shandong University (2012TS214).

The authors are grateful to the anonymous referee for a careful checking of the details and for helpful comments that improved this paper.

\section{References}

[1] S. M. Song and K. J. Waldron, Editors, Machines That Walk: The Adaptive Suspension Vehicle (MIT Press, Cambridge, 1989).

[2] M. Raibert, Legged robots that balance (MIT Press, Cambridge, 1986).

[3] K. Berns and W. Ilg, Mechanical construction and computer architecture of the fourlegged walking machine BISAM, ASME transactions on mechatronics, 4, (1999).

[4] C. Ridderstom and J. Ingvast, Quadruped posture control based on simple force distribution - a notion and a trial, IEEE Conferrnce on Intelligent Robots and Systems, Mad, Hawaii, USA, (2001).

[5] L. R. Palmer III and D. E. Orin, Quadrupedal running at high speed over uneven terrain, Proceedings of the IEEE/RSJ Conferrnce on Intelligent Robots and Systems, San Diego, USA, (2007).

[6] H. Kimura, Y. Fukuoka and A. H. Cohen, Adaptive dynamic walking of a quadruped robot on irregular terrain based on biological concepts, The International Journal of Robotics Research, 22, (2003).

[7] J. Chung, I. Park and J. Oh, On the Design and Development of a Quadruped Robot Platform, Advanced Robotics, 24, (2010).

[8] M. Raibert, K. Blankespoor, G. Nelson et al, BigDog, the Rough-Terrain Quadruped Robot, International Federation of Automation Control, Seoul, Korea, (2008).

[9] T. Kim, B. So, O. Kwon, and S. Park, The energy minimization algorithm using foot rotation for hydraulic actuated quadruped walking robot with redundancy, The 6th German Conference on Robotics, Munich, Germany, (2010).

[10] C. Semini, HyQ - Design and Development of a Hydraulically Actuated Quadruped Robot, $\mathrm{PhD}$ dissertation, University of Genoa, Italy, (2010).

[11] X. W. Rong, Y. B. Li, J. H. Ruan and H. J. Song, Kinematics analysis and simulation of a quadruped robot, Applied Mechanics and Materials, 26-28, (2010).

[12] H. J. Song, X.W. Rong, Y. B. Li and J. H. Ruan, Study on Kinematics-based Symbolic Computation-driven Quadruped Robot Gait Simulation System, Key Engineering Materials, 480-481, (2011).

[13] Y. Sakakibara, K. Kan, Y. Hosoda, M. Hattori, and M. Fujie, Foot trajectory for a quadruped walking machine, IEEE International Workshop on Intelligent Robots and Systems, Ibaraki, Japan, (1990).

[14] K. Y. Kim and J. H. Park, Ellipse-based leg-trajectory generation for galloping quadruped robots, Journal of Mechanical Science and Technology, 22, (2008).

[15] MSC.Software, MSC.ADAMS/VIEW advanced training guide (Tsinghua University Press, Beijing, 2004).

[16] N. C. Heglund and C. R. Taylor, Speed, stride frequency and energy cost per stride: how do they change with body size and gait? Journal of Experimental Biology, 138, (1988).
[17] N. C. Heglund, C. R. Taylor, and T. A. McMahon, Scaling Stride Frequency and Gait to Animal Size: Mice to Horses, Science, 4169, (1974).

[18] K. T. Stranga and K. Teudel, Explaining the scaling of transport costs: the role of stride frequency and stride length, Journal of Zoology, 221, (1990).

[19] C. X. Wang, Editor, Hydraulic control system (China Machine Press, Beijing, 2010).

[20] Y. J. Li, Aircraft Hydraulic Transmission and Control (Science Press, Beijing, 2009).
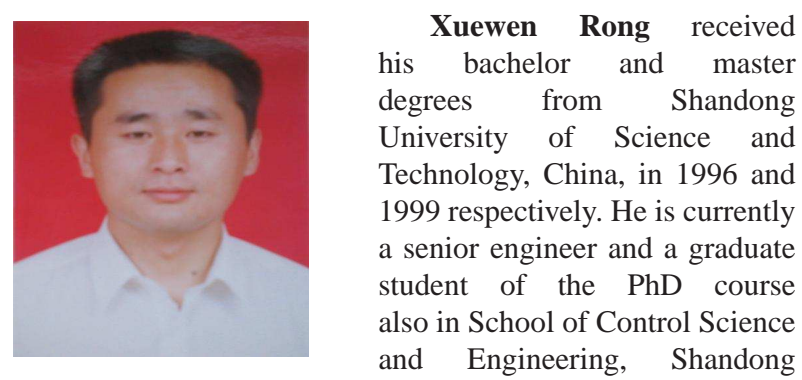

University, China. His research interests include robotics, mechatronics, hydraulic servo driving technology, etc.

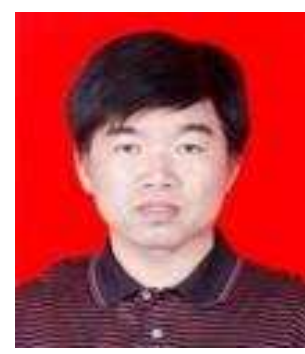

Yibin $\mathbf{L i}$ received his bachelor and doctor degrees from Tianjin University, China, in 1982 and 2006 respectively. $\mathrm{He}$ received his master degree from Shandong University of Science and Technology, China, in 1988. He is currently a professor and associate dean in School of Control Science and Engineering, Shandong University, China. His research interests include robotics, mechatronics, intelligent control, intelligent vehicles, etc.

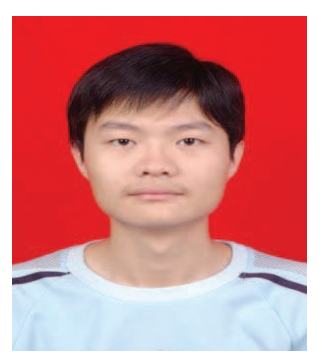

Jian Meng received his Bachelor degree from Shandong Jianzhu university, China, in 2009. He is now a doctoral student in the school of control science of engineering, Shandong university, China. $\mathrm{He}$ works on the control system of quadruped robot.

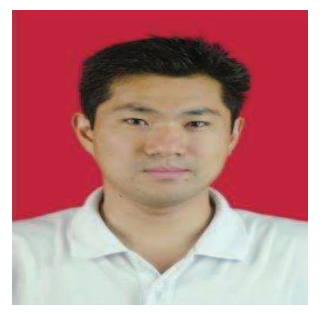

Li Bin received his B. Sc. and M. Sc. degrees at Shandong University, China, in 2002 and 2005 , respectively, and the $\mathrm{Ph}$. D. degree at Shandong University, China in 2012. His research interest covers algorithms for neural networks and gait planning of legged robots, etc. 